\title{
A symmetric DBI action theory and its applications to inflationary cosmology
}

\author{
Xiaokun Yang ${ }^{1, \mathrm{a}}$, Wu-Long $\mathrm{Xu}^{1, \mathrm{~b}}$, Yong-Chang Huang ${ }^{1,2, \mathrm{c}}$ \\ ${ }^{1}$ Institute of Theoretical Physics, Beijing University of Technology, Beijing 100022, China \\ ${ }^{2}$ Institute of Theoretical Physics, Jiangxi Normal University, Nanchang 330022, China
}

Received: 4 August 2021 / Accepted: 5 December 2021 / Published online: 24 December 2021

(C) The Author(s) 2021

\begin{abstract}
The Dirac-Born-Infeld (DBI) field theory in string theory is important and can provide the field of the universe's inflation. At the same time, it provides a causal mechanism for generating the original density perturbation, thereby providing the necessary density perturbation for existing the dense and sparse matter distributions of the universe. We deduce a symmetric DBI action, introduce it into inflationary cosmology to calculate various inflation parameters, further calculate the scalar perturbation spectrum and the tensor-scalar ratio, which are compared with Planck + WMAP9 + BAO data, the power spectrum predicted by the new general DBI inflation theory satisfies the CMB Experiment constraints, i.e., is consistent with the current theories and experimental observations. Consequently, the theory of this paper conforms to current experiments and is supplying the current theories, and also a new way of explaining the inflation of the universe.
\end{abstract}

\section{Introduction}

Current cosmology has two important discoveries, one is Hubble expansion, and the other is cosmic microwave background radiation. These two discoveries tell us that our universe is expanding, and it is uniform and flat [1-6]. Therefore the production mechanism of such a flat universe was proposed. For explaining the flatness of the universe, the horizon of the universe and the problem of magnetic monopoles, the most mainstream theory now is the theory of cosmic inflation. It means that the universe experienced a rapid acceleration expansion in the early stage of the creation, and the causal connection area rapidly expanded into a large area for forming an observable universe, and the non-flatness was

\footnotetext{
a e-mail: xkyang@emails.bjut.edu.cn

b e-mail: wlxu@emails.bjut.edu.cn (corresponding author)

${ }^{\mathrm{c}}$ e-mail: ychuang@bjut.edu.cn
}

smoothed. Moreover, the theory of cosmic inflation can provide the original perturbation, thus giving birth seeds to the galaxy structure. The cosmic inflation is usually realized by scalar fields [7-15]. We call this scalar field as inflation field. Inflation can be also realized purely geometrically, in particular in the Starobinsky $R+R^{2}$ model [16] which was historically the first one and which remains viable by now, in contrast to many models proposed later, in Refs. [7-15] in particular.

In the very early stage of the universe, the initial value of the potential energy $V(\varphi)$ of the inflation field $\varphi$ was very large, and the potential energy curve was very flat. This potential energy provides the energy of the universe's inflation. With the evolution of time and the change of the inflation field, the potential energy slowly decreases, and the scaling factor of the universe increases almost exponentially in this process, resulting in the universe inflation that we predict.

The general theory of cosmic inflation does not give the source of the scalar field. A major contribution of string theory is that the DBI field theory produced by string theory can provide the field of the universe's inflation [9, 10, 17-20]. This theory shows that the inflation of the universe is related to the motion of D-brane in the 6-dimensional compact submanifold of spacetime. It is the motion of a certain string that causes the scalar field to be generated, and it restricts the motion of the scalar field. This is an effective field theory explanation.

This explanation is based on string theory and is quite consistent in terms of the construction mechanism. When we integrate other dimensions to 4 dimensions, the DBI action will naturally be deduced. String theory also gives the behavior of cosmic inflation, so people can make general cosmological predictions based on a set of given background parameters [21]. This makes the brane universe inflation a good theory for exploring the universe's inflation. Therefore, it is possible to establish the universe evolution theory that satisfies various parameters and is consistent with the basic theory. 
There has been a lot of work in studying the inflation of the universe and understanding the inflation field [22-28] .

The DBI action can constructs the inflationary cosmology [29,30], which considers that $A d s_{5}$ space is related to a "throat"-shaped area. Then in this region, the radial coordinates of the moving $D_{3}-$ brane are taken as a scalar field in our universe. With the deformation of $A d s_{5}$ space, this throat-like area will also be deformed, then the moving $D_{3}-$ brane will be compressed, causing it to change accordingly [30]. The change of the radial coordinate of the $D_{3}-$ brane, that is, the movement of this scalar field, is affected by the distortion coefficient of the throat $[21,31]$.

When inflationary cosmology solves the problem of flatness, monopoles and the difficulty of horizon, it is natural that due to the quantum perturbation in the inflation process, it will produce seeds of large-scale structure for the universe. There are two sources of perturbation. One is the perturbation caused by the stretching of spacetime itself in inflation. This perturbation is embodied as a gravitational wave. We call it adiabatic perturbation or tensor perturbation [32], the other comes from the quantum perturbation caused by the scalar field that produces inflation, we call it scalar perturbation. The source of tensor perturbation is essentially different from scalar perturbation.

However, none of the current DBI theories considers the puzzle, for example the literature [26,33,34], of the conversion of potential energy and kinetic energy in equal rights. The problem, in other words, the conversion of potential energy and kinetic energy with each other in these actions can only be carried out under the first-order approximation, and the potential energy can not be completely converted to kinetic energy under high-order. Therefore, we want to give a symmetric DBI action, which enables the kinetic energy and potential energy in the action to be converted each other in equal rights. Since we used the conformal transformation when obtaining the symmetrical DBI action, the potential energy $V$ will be naturally generated from this transformation, instead of manually adding the potential energy term like the current general DBI action. This makes our theory be consistent.

The symmetric DBI action has the advantage of equal rights of kinetic energy and potential energy, so whether it can meet with the existing observational data is the main research point. After we deduce the symmetric DBI action by string theory, we introduce it into inflationary cosmology to calculate various inflation parameters, and calculate the scalar perturbation spectrum and tensor-scalar ratio, further compare them with Planck + WMAP9 + BAO data to verify the correctness of the new general DBI action. Whether it conforms to the observational data and whether it will violate the prediction of the standard DBI action, then we need to verify whether the symmetric DBI action can be driven by inflation and produce the vast universe we see now. And lots of DBI theories and their applications to different physics systems have been very well given out, e.g., see Refs. [3541].

In the second section, we derive a symmetric DBI Lagrangian for inflation. This Lagrangian contains various possible interaction potentials. Furthermore, we naturally derive the determinant of the induced metric, and then compare the symmetric DBI I Lagrangian with the current general DBI Lagrangian. In the third section, we obtained the cosmological equations and cosmological parameters deduced from the new general Lagrangian, and calculate the slowrolling parameters and e-ford number. In the fourth section, we calculate the power spectrum of the original perturbation under the linear perturbation, and calculate the form of the tensor-scalar ratio. In order to reflect the relationship between the original scalar perturbation and the inflation field, we use perturbation to directly calculate the magnitude of the perturbation amplitude to verify the true role of the inflation field in it. In the fifth section, we have selected different background fields and potential functions, and obtained the functional relationship diagrams between the tensor-scalar ratio index of the original scalar perturbation, and compared it with the background of the Planck + WMAP9 + BAO joint data. In the two cases of $N=50$ and $N=60$, the tensor-scalar ratio is reflected by different parameter groups, thus their correct properties are proved. In the sixth section, we show discussion. The final section is conclusion.

\section{New general DBI action}

In this section, we will start with string theory, consider the conformal transformation to derive a symmetric DBI action, and compare it with the current general DBI action.

Here we deduce a symmetric DBI Lagrangian, and find that the determinant of the metric can naturally include momentum energy and potential energy in equal rights.

The current general DBI Lagrangian is in the form of [4244]:

$\mathcal{L}=-f^{-1}(\varphi) \sqrt{1+f(\varphi) g^{\mu \nu} \partial_{\mu} \varphi \partial_{\nu} \varphi}-V(\varphi)+f^{-1}(\varphi)$

Obviously, in such a Lagrangian, the kinetic energy $\partial_{\mu} \varphi \partial_{\nu} \varphi$ and the potential energy $V(\varphi)$ have the puzzle of unequal rights. Therefore, we need to consider a symmetric DBI action and solve the puzzle for the current general DBI action. When establishing this symmetric DBI action, we consider that the field that promotes the inflation of the universe exists in a $(1+3)$ dimensional spacetime and a 6 -dimensional curly spacetime. And regarding the $(1+3)$ dimensional world body as a particle migrating along the 
radial $r$ and passing through the 6-dimensional curly spacetime $[17,18,45,46,48]$. Then the corresponding line element can be expressed as $[22,26,33,34]$ :

$d s_{10}^{2}=h^{2}(r) d s_{4}^{2}+h^{-2}(r)\left(d r^{2}+r^{2} d s_{y_{5}}^{2}\right)$.

Take the metrics of $d s_{4}^{2}$ and $d s_{y_{5}}^{2}$ as $a_{\mu v}(x)$ and $a_{a b}(y)$ respectively and do conformal transformation on them:

$a_{\mu \nu}^{\prime}(x)=W_{1}(r) a_{\mu \nu}(x)$

$a_{a b}^{\prime}(y)=W_{1}(r) a_{a b}(y)$.

Then, the metric matrixes can be written as:

$\begin{aligned} G & =\left[\begin{array}{ccc}h^{2}(r) a_{\mu \nu}(x) & & \\ & h^{-2}(r) & \\ & & h^{-2}(r) r^{2} a_{a b}(y)\end{array}\right] \\ G^{\prime} & =\left[\begin{array}{lll}h^{2}(r) W_{1}(r) a_{\mu \nu}(x) & & \\ & h^{-2}(r) & \\ & & h^{-2}(r) W_{1}(r) r^{2} a_{a b}(y)\end{array}\right] .\end{aligned}$

Under the conformal transformations (3) and (4), the induced world volumes elements are invariant, namely:

$\sqrt{-\operatorname{det} G} d^{4} x=\sqrt{-\operatorname{det} G^{\prime}} d^{4} x^{\prime}$

Then, integrating Eq. (7), we deduce a general action:

$S=\chi \int d^{4} x \sqrt{-\operatorname{det} G}=\chi \int d^{4} x^{\prime} \sqrt{-\operatorname{det} G^{\prime}}$

where $\chi$ is an invariant parameter with dimension so that the product of $\chi$ and the world body integral is the general action, and the general action (8) is invariant under the conformal transformation.

Therefore, in string theory and $\mathrm{M}$ theory, the induced metric in our four-dimensional spacetime is:

$$
\begin{aligned}
G_{\alpha \beta}= & h^{2}(r) W_{1}(r) a_{\mu \nu}(x) \frac{\partial x^{\mu}}{\partial x^{\alpha}} \frac{\partial x^{\nu}}{\partial x^{\beta}} \\
& +h^{-2}(r) \frac{\partial r}{\partial x^{\alpha}} \frac{\partial r}{\partial x^{\beta}} \\
& +h^{-2}(r) W_{1}(r) r^{2} a_{a b}(y) \frac{\partial x^{a}}{\partial x^{\alpha}} \frac{\partial x^{b}}{\partial x^{\beta}} .
\end{aligned}
$$

Since $y$ has nothing to do with $x$ in $(1+3)$ dimensional spacetime, where $\mu, v, \alpha, \beta(=0,1,2,3), a, b(=5,6,7$, $8,9), x^{4}=r$. Thus, the third term in $G_{\alpha \beta}$ satisfies

$$
h^{-2}(r) W_{1}(r) r^{2} a_{a b}(y) \frac{\partial x^{a}}{\partial x^{\alpha}} \frac{\partial x^{b}}{\partial x^{\beta}}=0
$$

Define $W_{1}(r)=1+W_{11}(r)$, where $W_{11}(r)$ is also a general function of $r$, then the inducted metric can be written as:

$G_{\alpha \beta}=h^{2}(r) g_{\alpha \beta}+h^{2}(r) W_{11}(r) g_{\alpha \beta}+h^{-2}(r) \frac{\partial r}{\partial x^{\alpha}} \frac{\partial r}{\partial x^{\beta}}$,

$W_{11}(r)$ is a general function.

Therefore, the symmetric DBI action on the $D_{3}-$ brane can be written as:

$S_{D B I L}$

$$
\begin{aligned}
= & -T \int d^{4} \sigma \sqrt{-\operatorname{det}\left[h^{2}(r) g_{\alpha \beta}+h^{2}(r) W_{11}(r) g_{\alpha \beta}+h^{-2}(r) \frac{\partial r}{\partial x^{\alpha}} \frac{\partial r}{\partial x^{\beta}}\right]} \\
= & -T \int d^{4} \sigma h^{4}(r) \\
& \times \sqrt{-\operatorname{detg}_{\alpha \lambda}} \sqrt{\operatorname{det}\left[\delta_{\beta}^{\lambda}\left(1+W_{11}(r)\right)+h^{-4}(r) g^{\lambda \tau} \frac{\partial r}{\partial x^{\tau}} \frac{\partial r}{\partial x^{\beta}}\right]} .
\end{aligned}
$$

We can get the string tension as:

$T_{p_{d}}=\frac{1}{g_{s}(2 \pi)^{d}\left(\alpha^{\prime}\right)^{2}}$.

So, for the $D_{3}-$ brane,

$T_{p_{3}}=\frac{1}{g_{s}(2 \pi)^{3}\left(\alpha^{\prime}\right)^{2}}$.

It is a function of string coupling strength $g_{s}$, so we can define inflationary scalar field $\varphi=r \sqrt{T_{p_{3}}}$.

Then, our DBI action can be written as:

$$
\begin{aligned}
S_{D B I L}= & -T_{p_{3}} \int d^{4} \sigma h^{4}(\varphi) \sqrt{-\operatorname{detg} \alpha \lambda} \\
& \times \sqrt{\operatorname{det}\left[\delta_{\beta}^{\lambda}\left(1+W_{11}(\varphi)\right)+h^{-4}(\varphi) g^{\lambda \tau} \partial_{\tau} \varphi \partial_{\beta} \varphi\right]} .
\end{aligned}
$$

Further, the action of DBI is written as:

$$
\begin{aligned}
S_{D B I L}= & -T_{p_{3}} \int d^{4} \sigma h^{4}(\varphi) \sqrt{-\operatorname{detg} \alpha \lambda} \\
& \times \sqrt{\operatorname{det}\left[\delta_{\beta}^{\lambda}\left(1+W_{11}(\varphi)\right)+h^{-4}(\varphi) g^{\lambda \tau} \partial_{\tau} \varphi \partial_{\beta} \varphi\right]} \\
= & -\int d^{4} \sigma f^{-1}(\varphi) \sqrt{-\operatorname{detg} \alpha \lambda} \\
& \times \sqrt{\operatorname{det}\left[\delta_{\beta}^{\lambda}\left(1+W_{11}(\varphi)\right)+f(\varphi) g^{\lambda \tau} \partial_{\tau} \varphi \partial_{\beta} \varphi\right]},
\end{aligned}
$$

where

$f(\varphi)=\frac{1}{T_{p_{3}} h^{4}(\varphi)}$ 
When taking $\sqrt{-\operatorname{detg} g_{\alpha}}$ to the invariant volume element of the integral (16) and adding an integral scalar term $T_{p_{3}} \int d^{4} \sigma h^{4}(\varphi) \sqrt{-\operatorname{det} g_{\alpha \lambda}}=\int d^{4} \sigma \sqrt{-\operatorname{detg} \alpha \lambda} f^{-1}(\varphi)$ to (16), we finally obtain a symmetric DBI Lagrangian.

$$
\begin{aligned}
& \mathcal{L}_{D B I L} \\
& =-f^{-1}(\varphi) \sqrt{\operatorname{det}\left[\delta_{\beta}^{\lambda}\left(1+W_{11}(\varphi)\right)+f(\varphi) g^{\lambda \tau} \partial_{\tau} \varphi \partial_{\beta} \varphi\right]} \\
& \quad+f^{-1}(\varphi) .
\end{aligned}
$$

The $f(\varphi)$ can be regarded as the background scalar field of the universe.

Because in the earliest period of the universe, all kinds of matter fields did not appear in the universe, but at this time the gravitational field and scalar field had already begun to act, so other types of type IIB fields did not appear in the action. Since $W_{11}(\varphi)$ is an arbitrary function, we define $W_{11}(\varphi)=f(\varphi) K V(\varphi)(K$ is a function of $\varphi)$. Under linear approximation, the Eq. (18) is rewritten as:

$$
\begin{aligned}
\mathcal{L}_{D B I L}= & -f^{-1}(\varphi) \sqrt{1+f(\varphi)\left[g^{\alpha \beta} \partial_{\alpha} \varphi \partial_{\beta} \varphi+K(\varphi) V(\varphi)\right]} \\
& +f^{-1}(\varphi) .
\end{aligned}
$$

The Eq. (19) uses a parameter to ensure that the kinetic energy item $g^{\alpha \beta} \partial_{\alpha} \varphi \partial_{\beta} \varphi$ is in the same equal position as the potential energy. This is the basic Physical requirements and general actual physical systems. Because if the system cannot naturally contain kinetic energy and potential energy, the system is not a general actual physical system. Therefore, it can be found that the conformal transformation is a very useful transformation, which has an important physical meaning relative to the curl factor $h$, which just keeps the kinetic energy and potential energy always in equal rights each other.

The Eq. (19) can be linearly approximated to the current general DBI Lagrangian. Taking the linear approximation of Lagrangian, we have:

$\mathcal{L}_{D B I L}=-\frac{1}{2} g^{\alpha \beta} \partial_{\alpha} \varphi \partial_{\beta} \varphi-\frac{1}{2} K(\varphi) V(\varphi)$.

Refer to the current general DBI Lagrangian (1) given by [42-44].The potential energy $V$ in this Lagrangian (1) looks as if it was directly added manually, and the kinetic energy and the potential energy are not in equal rights, because

$$
\begin{aligned}
\mathcal{L}_{D B I}= & \left.-\frac{1}{2} g^{\alpha \beta} \partial_{\alpha} \varphi \partial_{\beta} \varphi-V(\varphi)\right) \\
& +\frac{1}{8} f(\varphi)\left(g^{\alpha \beta} \partial_{\alpha} \varphi \partial_{\beta} \varphi\right)^{2} \\
& -\frac{1}{16} f^{2}(\varphi)\left(g^{\alpha \beta} \partial_{\alpha} \varphi \partial_{\beta} \varphi\right)^{3} \cdots .
\end{aligned}
$$

Do a linear approximation to this Lagrangian, one can get:

$\mathcal{L}_{D B I}=-\frac{1}{2} g^{\alpha \beta} \partial_{\alpha} \varphi \partial_{\beta} \varphi-V(\varphi)$

If in a physical system, the kinetic energy and the potential energy cannot be transformed each other in equal rights, anyone can believe that this is not a real physical situation, and this symmetric DBI action can give the transformation of kinetic energy and potential energy in equal rights.

The comparing formula (20) with Eq. (22), if we take $K=2$, the Eq. (20) will be simplified to (22). This shows that in the linear approximation, or equivalently in the nonrelativistic limit of D-brane's motion, Lagrangian (1) is similar to our symmetric DBI Lagrangian.

Note that Eq. (19) is symmetric than Eq. (1), because Eq. (19) shows that potential energy can be converted to kinetic energy in equal rights, and the potential energy naturally emerges from the Lagrangian, without manual adding to Eq. (22) is only a linear approximation of the special DBI action, and the Eq. (19) is the symmetric Lagrangian of the DBI action. The potential energy in the formula of (1) cannot be directly converted into kinetic energy in equal rights, which destroys the basic physical principles. The symmetric DBI Lagrangian (19) is different from previous work. Equation (19) reveals the basic physical meaning of the potential energy generated by $D_{3}$-brane. This section solves the puzzle of conversion of potential energy and kinetic energy in equal rights.

\section{Inflationary cosmology and inflationary parameters}

In this section, we will use the new DBI action to generate the inflationary cosmological action, get the density and momentum driven by the inflation field in the early universe, and calculate the inflation parameter and e-ford number. It is worth noting that is although the original DBI action is included when $K$ is a function of $\varphi$, in the following, we consider the cases with constant $\mathrm{K}$, for simplicity.

We apply this action to the dynamics of the inflationary universe [47]:

$$
\begin{aligned}
S= & \int d x^{4} \sqrt{-g}\left[\frac{1}{\kappa^{2}} R-f^{-1}(\varphi)\right. \\
& \left.\times \sqrt{1+f(\varphi)\left[g^{\alpha \beta} \partial_{\alpha} \varphi \partial_{\beta} \varphi+K V(\varphi)\right]}+f^{-1}(\varphi)\right],
\end{aligned}
$$

where $R$ is a Ricci scalar, then the Einstein equation obtained from this action is: 
$G_{\mu \nu}=\kappa^{2} T_{\mu \nu}$.

$T_{\mu \nu}$ comes from the DBI action:

$$
\begin{aligned}
T_{\mu \nu}= & \frac{2 \partial\left[-f^{-1}(\varphi) \sqrt{1+f(\varphi)\left[g^{\alpha \beta} \partial_{\alpha} \varphi \partial_{\beta} \varphi+K V(\varphi)\right]}\right]}{\partial g^{\mu \nu}} \\
& -g_{\mu \nu}\left[-f^{-1}(\varphi) \sqrt{1+f(\varphi)\left[g^{\alpha \beta} \partial_{\alpha} \varphi \partial_{\beta} \varphi+K V(\varphi)\right]}\right. \\
& \left.+f^{-1}(\varphi)\right] .
\end{aligned}
$$

Therefore, different metric needs to be selected to find the corresponding Einstein field equation.

Select FRW metric:

$d s^{2}=d t^{2}-a^{2}(t)\left(\delta_{i j}+k \frac{x_{i} x_{j}}{1-k r^{2}}\right) d x^{i} d x^{j}$,

where $k=-1,0,1$. Using Eq. (25), the density and momentum of matter can be obtained as:

$$
\begin{aligned}
\rho= & -\frac{\dot{\varphi}^{2}}{\sqrt{1+f \dot{\varphi}^{2}+K f V}} \\
& -f^{-1}+f^{-1} \sqrt{1+f \dot{\varphi}^{2}+K f V} \\
p= & \frac{\partial_{j} \varphi \partial^{j} \varphi}{3 \sqrt{1+f \dot{\varphi}^{2}+K f V}} \\
& +f^{-1}-f^{-1} \sqrt{1+f \dot{\varphi}^{2}+K f V}
\end{aligned}
$$
tion:

Solving the Einstein equation, we get the Friedmann equa-

$$
\begin{aligned}
H^{2}= & \frac{\kappa^{2}}{3}\left(-\frac{\dot{\varphi}^{2}}{\sqrt{1+f \dot{\varphi}^{2}+K f V}}\right. \\
& \left.-f^{-1}+f^{-1} \sqrt{1+f \dot{\varphi}^{2}+K f V}\right)
\end{aligned}
$$

Doing variation on the action (23), we get the equation of motion:

$$
\begin{gathered}
\frac{\ddot{\varphi}}{\left(1+f \dot{\varphi}^{2}+K f V\right)^{\frac{3}{2}}}+\frac{3 H \dot{\varphi}}{\left(1+f \dot{\varphi}^{2}+K f V\right)^{\frac{1}{2}}} \\
=-f^{\prime} f^{-2}+f^{\prime} f^{-2}\left(1+f \dot{\varphi}^{2}+K f V\right)^{\frac{1}{2}} \\
-\frac{1}{2} \frac{f^{\prime} f^{-1} \dot{\varphi}^{2}+K f^{\prime} f^{-1} V+K V^{\prime}}{\left(1-f \dot{\varphi}^{2}+K f V\right)^{\frac{1}{2}}}
\end{gathered}
$$

If we consider the slow roll approximation $\dot{\varphi}^{2} \ll 1$ and $\ddot{\varphi} \ll|3 H \dot{\varphi}|$, the energy density of the DBI field and the equation of motion will become:

$$
\begin{aligned}
\rho & =-f^{-1}+f^{-1} \sqrt{1+K f V} \\
H^{2} & =\frac{\kappa^{2}}{3} \frac{1}{f}(\sqrt{1+K f V}-1)
\end{aligned}
$$

$3 H \dot{\varphi}=-f^{\prime-2} \sqrt{1+K f V}+f^{\prime-2}+\frac{1}{2} K f^{\prime-1} V-\frac{1}{2} K V^{\prime}$.

Then we can get the slow roll parameter $\epsilon \equiv-\frac{\dot{H}}{H^{2}}, \eta \equiv$ $-\frac{\ddot{H}}{H \dot{H}}$, namely:

$$
\begin{aligned}
\epsilon \equiv-\frac{\dot{H}}{H^{2}}= & \frac{\kappa^{2}}{18 H^{4}}(1+K f V)^{-\frac{1}{2}} \\
& \times\left[f^{-2} f^{\prime}-f^{-2} f^{\prime} \sqrt{1+K f V}\right. \\
& \left.+\frac{1}{2} K f^{-1} f^{\prime} V-\frac{1}{2} K V^{\prime}\right]^{2} \\
= & \frac{\kappa^{2}}{18 H^{4}}(1+K f V)^{-\frac{1}{2}} Y^{2} \\
\eta= & -\frac{Y}{3 H^{2}}\left(\frac{2 Y^{\prime}}{Y}-\frac{Y}{H^{2}}+\frac{1}{2} \frac{K f^{\prime} V+K f V^{\prime}}{1+K f V}\right),
\end{aligned}
$$

where

$Y=-f^{\prime-2} \sqrt{1+K f V}+f^{\prime-2}+\frac{1}{2} K f^{\prime-1} V-\frac{1}{2} K V^{\prime}$.

The inflation occurs under the conditions of $\{\epsilon, \eta\}<1$; once these slow-rolling parameters reach 1 , the inflation phase ends. The e-ford number during the inflation period is defined as:

$N=\int_{t_{h c}}^{t_{f}} H d t$

For our model, the e-ford number during slow scrolling can be expressed as:

$$
\begin{aligned}
N & =\int_{\varphi_{h c}}^{\varphi_{f}} \frac{3 H^{2}}{3 H \dot{\varphi}} d \varphi \\
& =\kappa^{2} \int_{\varphi_{h c}}^{\varphi_{f}} \frac{1-\sqrt{1+K f V}}{f^{\prime-1} \sqrt{1+K f V}-f^{\prime-1}+\frac{1}{2} K f^{\prime} V-\frac{1}{2} K f V^{\prime}} d \varphi,
\end{aligned}
$$

where $\varphi_{h c}$ represents the field value when the observed universe scale crosses the Hubble horizon during inflation, and $\varphi_{f}$ is the field value when the universe exits the inflation phase.

\section{Inflation Perturbation}

In this section, we will study the theory of linear perturbations by the new DBI action.

In many different methods, according to the choice of the metric that characterizes the cosmic perturbation, we choose the longitudinal metric. The scalar metric perturbation of the 
FRW background is given by the literature [48-50]:

$d s^{2}=(1+2 \Phi) d t^{2}-a^{2}(t)(1-2 \Psi) \delta_{i j} d x^{i} d x^{j}$,

where $a(t)$ is the scale factor of the universe, metric perturbations $\Phi=\Phi(x, t)$ and $\Psi=\Psi(x, t)$ are gauge invariant variables. All perturbations behave like plane waves $e^{i k x}$ in space, where $k$ is the wave number. Through Einstein's field equations, any perturbation of the metric will cause perturbations of the energy-momentum tensor. For the perturbed metric (39), the perturbed Einstein field equation can be obtained:

$$
\begin{aligned}
& 6 H(H \Phi+\dot{\Psi})-\frac{2 k^{2}}{a^{2}} \\
& =\kappa^{2} f^{\prime} f^{-2}\left(1-\frac{1}{\sqrt{1+f \dot{\varphi}^{2}+K f V}}\right) \delta \varphi \\
& +\kappa^{2} \frac{f^{\prime} \dot{\varphi}^{2} \delta \varphi+f\left(\dot{\varphi} \delta \dot{\varphi}+\dot{\varphi}^{2} \Phi\right)+K\left(f^{\prime} V+f V^{\prime}\right) \delta \varphi}{\left(1+f \dot{\varphi}^{2}+K f V\right)^{\frac{3}{2}}}
\end{aligned}
$$

$$
\begin{aligned}
2 \ddot{\Psi} & +6 H(H \Phi+\dot{\Psi})+2 H \dot{\Phi} \\
& +4 H \Phi+\frac{2}{3 a^{2}} k^{2}(\Phi-\Psi) \\
= & -\kappa^{2} f^{\prime} f^{-2}\left(1-\frac{1}{\sqrt{1+f \dot{\varphi}^{2}+K f V}}\right) \delta \varphi \\
& -\kappa^{2} \frac{f^{\prime} \dot{\varphi}^{2} \delta \varphi+f\left(\dot{\varphi} \delta \dot{\varphi}+\dot{\varphi}^{2} \Phi\right)+K\left(f^{\prime} V+f V^{\prime}\right) \delta \varphi}{\left(1+f \dot{\varphi}^{2}+K f V\right)^{\frac{3}{2}}}
\end{aligned}
$$

$$
\begin{aligned}
2 \ddot{\Psi}+ & 6 H(H \Phi+\dot{\Psi})+2 H \dot{\Phi} \\
& +4 H \Phi+\frac{2}{3 a^{2}} k^{2}(\Phi-\Psi) \\
= & -\kappa^{2} f^{\prime} f^{-2}\left(1-\frac{1}{\sqrt{1+f \dot{\varphi}^{2}+K f V}}\right) \delta \varphi \\
& -\kappa^{2} \frac{f^{\prime} \dot{\varphi}^{2} \delta \varphi+f\left(\dot{\varphi} \delta \dot{\varphi}+\dot{\varphi}^{2} \Phi\right)+K\left(f^{\prime} V+f V^{\prime}\right) \delta \varphi}{\left(1+f \dot{\varphi}^{2}+K f V\right)^{\frac{3}{2}}}
\end{aligned}
$$

$$
\begin{aligned}
& H \Phi+\dot{\Psi}=-\frac{\kappa^{2} H^{2}}{\sqrt{1+f \dot{\varphi}^{2}+K f V}} \dot{\varphi} \delta \varphi \\
& \Phi-\Psi=0 .
\end{aligned}
$$

Performing variation on the equation of motion (23) for $\varphi$, we get:

$$
\begin{aligned}
& \frac{\delta \ddot{\varphi}}{1+f \dot{\varphi}^{2}+K f V} \\
& -\ddot{\varphi} \frac{f^{\prime} \dot{\varphi}^{2} \delta \varphi+f\left(\dot{\varphi} \delta \dot{\varphi}+\dot{\varphi}^{2} \Phi\right)+K\left(f^{\prime} V+f V^{\prime}\right) \delta \varphi}{\left(1+f \dot{\varphi}^{2}+K f V\right)^{2}} \\
& +\left(f^{\prime \prime} f^{-2}-2 f^{\prime 2} f^{-3}\right) \\
& \times\left(1+f \dot{\varphi}^{2}+\frac{1}{2} K f V+\sqrt{1+f \dot{\varphi}^{2}+K f V}\right) \delta \varphi
\end{aligned}
$$

$$
\begin{aligned}
& +3 H \delta \dot{\varphi}+\dot{\varphi}(\dot{\Phi}+3 \dot{\Psi}) \\
& -K f^{\prime} f^{-2}\left(-f^{\prime} V+f V^{\prime}\right) \delta \varphi-\frac{1}{2} f^{\prime} f^{-1} \\
& \left(\dot{\varphi} \delta \dot{\varphi}+\dot{\varphi}^{2} \Phi\right) \\
& +\frac{f^{\prime 2} f^{-2} \dot{\varphi}^{2} \delta \varphi+f^{\prime} f^{-1}\left(\dot{\varphi} \delta \dot{\varphi}+\dot{\varphi}^{2} \Phi\right)+K f^{\prime} f^{-2}\left(-f^{\prime} V+f V^{\prime}\right) \delta \varphi}{2 \sqrt{1+f \dot{\varphi}^{2}+K f V}} \\
& -\frac{1}{2} K V^{\prime \prime} \delta \varphi=0 .
\end{aligned}
$$

In order to obtain scalar perturbations and tensor perturbations in our model, it is sufficient to consider the slowrolling approximation of large-scale $k \ll a H$. In this case $\ddot{\Phi}, \ddot{\Psi}, \dot{\Phi}, \dot{\Psi}$ can be ignored (see [51-54]). Therefore, in a large range, the perturbation equation of motion can be written in the following form:

$3 H \delta \dot{\varphi}+\mathcal{K} \delta \varphi=0$,

and

$\dot{\varphi} \delta \dot{\varphi}+\dot{\varphi}^{2} \Phi=0$,

where

$$
\begin{aligned}
\mathcal{K}=( & \left.f^{\prime \prime} f^{-2}-2 f^{\prime 2} f^{-3}\right)\left(1+\frac{1}{2} K f V+\sqrt{1+K f V}\right) \\
- & K f^{\prime} f^{-2}\left(f^{\prime} V+f V^{\prime}\right)\left(1-\frac{1}{2}(1+K f V)^{-\frac{1}{2}}\right) \\
& -\frac{1}{2} K f^{\prime 2} f^{-2} V \\
& +\frac{1}{2} K f^{\prime \prime} f^{-1} V+\frac{1}{2} K f^{-1} f^{\prime} V^{\prime}+\frac{1}{2} K V^{\prime \prime} .
\end{aligned}
$$

For small scale perturbations, since the spatial distance is very small, the space can be regarded as flat at this time. At this time, the perturbation should be a quantum field theory plane wave solution in a flat spacetime. Therefore, the perturbation of the co-moving curvature on the super-horizon scale is almost constant:

$d \ln k(\varphi)=d N(\varphi)$.

Therefore, the perturbation is decomposed into two parts, one part is parallel to the cosmic brane trajectory, called adiabatic perturbation or curvature perturbation (if there is only one scalar field during inflation, we will deal with this type of perturbation [55-58]); the other part is the perturbation orthogonal to the trajectory, which we call entropy perturbation or scalar perturbation $[55,56,59,60]$.

In order to obtain the explicitness of the perturbation field $\delta \varphi$, we introduce the function $\mathcal{A}$ :

$\mathcal{A}=\frac{V^{\prime}}{V} \delta \phi$. 
Then, we can rewrite the Eq. (46) as:

$$
\begin{aligned}
& \frac{\mathcal{A}^{\prime}}{\mathcal{A}}=\frac{V^{\prime}}{V}-\frac{V^{\prime \prime}}{V^{\prime}} \\
& +\frac{\mathcal{K}}{-f^{\prime-2} \sqrt{1+K f V}+f^{\prime-2}+\frac{1}{2} K f^{\prime-1} V-\frac{1}{2} K V^{\prime}} .
\end{aligned}
$$

We can get:

$$
\mathcal{A}=C \exp \left(\int \frac{\mathcal{A}^{\prime}}{\mathcal{A}} \delta \phi\right)
$$

where $C$ is the integral constant.

So we can solve the perturbation field $\delta \phi$

$$
\begin{aligned}
\delta \phi= & \frac{C V^{\prime}}{V} \exp \left(\int \left[\frac{V^{\prime}}{V}-\frac{V^{\prime \prime}}{V^{\prime}}\right.\right. \\
& \left.\left.+\frac{\mathcal{K}}{-f^{\prime-2} \sqrt{1+K f V}+f^{\prime-2}+\frac{1}{2} K f^{\prime-1} V-\frac{1}{2} K V^{\prime}}\right] d \phi\right) .
\end{aligned}
$$

We can get the following expressions for the density perturbation amplitude:

$$
\begin{aligned}
A_{s}^{2}= & \frac{k^{3} C}{2 \pi^{2}} \frac{V^{\prime 2}}{V^{2}} \exp \left(\int \left[\frac{V^{\prime}}{V}-\frac{V^{\prime \prime}}{V^{\prime}}\right.\right. \\
& \left.\left.+\frac{\mathcal{K}}{-f^{\prime-2} \sqrt{1+K f V}+f^{\prime-2}+\frac{1}{2} K f^{\prime-1} V-\frac{1}{2} K V^{\prime}}\right] d \varphi\right) .
\end{aligned}
$$

Further obtain the spectrum index of the original scalar perturbation:

$$
\begin{aligned}
n_{s}-1=\frac{d \ln A_{s}^{2}}{d N(\varphi)}=\frac{1}{3 H^{2}}\left[\frac{V^{\prime}}{V}-\frac{V^{\prime \prime}}{V^{\prime}}\right. & \\
& \left.+\frac{\mathcal{K}}{-f^{\prime-2} \sqrt{1+K f V}+f^{\prime-2}+\frac{1}{2} K f^{\prime-1} V-\frac{1}{2} K V^{\prime}}\right] .
\end{aligned}
$$

Similarly, when away from the Hubble radius, the tensor perturbation amplitude in this mode is:

$$
A_{T}^{2}=\left.\frac{4 \kappa^{2}}{25 \pi} H^{2}\right|_{k=a H}=\frac{4 \kappa^{4}}{75} \frac{1}{f}(\sqrt{1+K f V}-1) .
$$

The ratio between the tensor perturbation amplitude and the scalar perturbation amplitude (tensor-scalar ratio $r$ ) is another important parameter, which is given by:

$$
\begin{aligned}
r= & \frac{A_{T}^{2}}{A_{s}^{2}}=\frac{8 \kappa^{4}}{75 k^{3} C} \frac{V^{2}(\sqrt{1+K f V}-1)}{V^{\prime 2} f} \\
& \times \exp \left(-\int\left[\frac{V^{\prime}}{V}-\frac{V^{\prime \prime}}{V^{\prime}}\right.\right. \\
& \left.\left.+\frac{\mathcal{K}}{-f^{\prime} f^{-2} \sqrt{1+K f V}+f^{\prime} f^{-2}+\frac{1}{2} K f^{\prime} f^{-1} V-\frac{1}{2} K V^{\prime}}\right] d \varphi\right) .
\end{aligned}
$$

\section{Constraints of observation on the model}

In the cosmological equation of the model, the new symmetrical DBI action has two functions, which play an important role in the dynamics of the model. They are $f(\varphi)$ and $V(\varphi) . f(\varphi)$ is given by the curl factor. In pure $A d s_{5}, f(\varphi)$ takes a simple form, namely $f(\varphi)=\beta \varphi^{-4}$. On the other hand, the reference [61] introduced another function of $f(\varphi)$ as $f(\varphi)=\beta e^{-\kappa \varphi}$. Therefore, we divide this section into two subsections. One has $f(\varphi)=\beta \varphi^{-4}$ and the other has $f(\varphi)=\beta e^{-\kappa \varphi}$. Then, we conduct the research by choosing the form of potential energy.

\section{$5.1 f(\varphi)=\beta \varphi^{-4}$}

For this type of $f(\varphi)$, we consider two types of potential: $V(\varphi)=\frac{\sigma}{2} \varphi^{2}$ quadratic and quartic potential $V(\varphi)=\frac{\sigma}{4} \varphi^{4}$. We observe that the parameter $K$ can be absorbed into the potential function $V$. Then, we redefine $\sigma^{\prime}=K \sigma$, then the parameter we have considered becomes $\left\{\beta, \sigma^{\prime}\right\}$. Next, we obtain some constraints on the model parameters by analyzing these parameters in the context of Planck + WMAP9 + BAO data.

$$
V(\varphi)=\frac{\sigma}{2} \varphi^{2}
$$

We consider the quadratic potential $V(\varphi)=\frac{\sigma}{2} \varphi^{2}$.

We introduce $\epsilon \equiv \frac{\dot{H}}{H}=1$ (corresponding to the end of the inflation), and we get $\varphi_{f}$. Then, by substituting the result into $N=\int_{t_{h c}}^{t_{f}} H d t$, we get $\varphi_{h c}$. By substituting $\varphi_{h c}$ into $n_{s}-1$ and $r=\frac{A_{T}^{2}}{A_{s}^{2}}$, we draw a relationship between the tensorscalar ratio and the spectral index. In the context of Planck + WMAP9 + BAO joint data, the following figure is drawn for $N=50$ and $N=60$ (Fig. 1):

As shown in Fig. 1, for some parameter combinations of $\left\{\sigma^{\prime}, \beta\right\}$, the model is compatible with Planck + WMAP9 + BAO data.

In Fig. 1 when the parameter group $\left\{\sigma^{\prime}, \beta\right\}$ take $\{0.8835$, $9.5547\}$, the model is at $N=50$. It is not compatible with Planck + WMAP9 + BAO data. When $N=60$, the model can be compatible with $67 \% \mathrm{CL}$ of Planck + WMAP9 + BAO data. When the parameter group $\left\{\sigma^{\prime}, \beta\right\}$ in the figure is taken as $\{1.1440,0.7592\}$, when $N=50$, the model can match Planck + WMAP9 + The $67 \%$ CL of the BAO data, which is compatible. When $N=60$, the model can be compatible with the $95 \% \mathrm{CL}$ of the Planck + WMAP9 + BAO data. When the parameter group $\left\{\sigma^{\prime}, \beta\right\}$ in the figure is taken as $\{1.4134,3.5523\}$, when $N=50$, the model can match Planck + WMAP9 + The 95\% CL of the BAO data, which is compatible. When $N=60$, the model can be compatible with the $95 \%$ CL of the Planck + WMAP9 + BAO data. 
Fig. 1 Relationships between the tensor-scalar ratio $r$ and the spectral index $n_{s}$. The blue, green, orange lines correspond to parameters, $\left\{\sigma^{\prime}, \beta\right\}=$ $\{0.8835,9.5547\}$, $\{1.1440,0.7592\}$,

$\{1.4134,3.5523\}$ respectively for $V(\varphi)=\frac{\sigma}{2} \varphi^{2}$

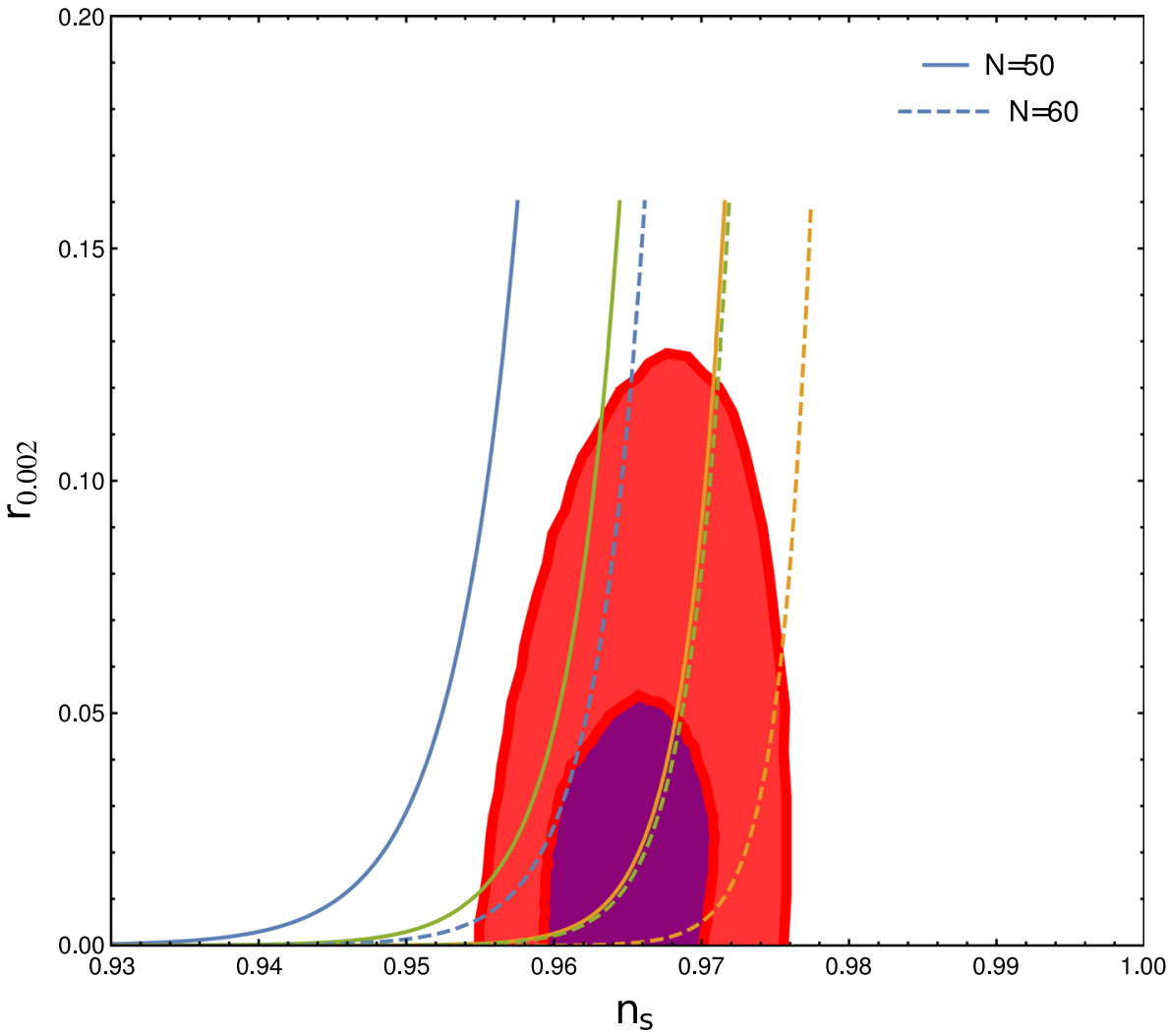

In the first step, we consider the quadratic potential $V(\varphi)=\frac{\sigma}{2} \varphi^{2}$.

We introduce $\epsilon \equiv \frac{\dot{H}}{H}=1$ (corresponding to the end of the inflation), and we get $\varphi_{f}$. Then, by substituting the result into $N=\int_{t_{h c}}^{t_{f}} H d t$, we get $\varphi_{h c}$. By substituting $\varphi_{h c}$ into $n_{s}-1$ and $r=\frac{A_{T}^{2}}{A_{s}^{2}}$, we draw a relationship between the tensorscalar ratio and the spectral index. In the context of Planck + WMAP9 + BAO joint data, the following figure is drawn for $N=50$ and $N=60$ (Fig. 2).

In the Fig. 2, parameter group $\left\{\sigma^{\prime}, \beta\right\}$ takes $\{1.1405,7.3919\}$, the model is at $N=50$. It is not compatible with Planck + WMAP9 + BAO data. When $N=60$, the model can be compatible with $95 \%$ CL of Planck + WMAP9 + BAO data. When the parameter group $\left\{\sigma^{\prime}, \beta\right\}$ is taken as $\{1.1141,1.0673\}$ in the figure, that the model can be compared with Planck + WMAP9 + The $67 \%$ CL of the BAO data is compatible. When $N=60$, that the model can be compatible with the $95 \% \mathrm{CL}$ of the Planck + WMAP9 + BAO data. When the parameter group $\left\{\sigma^{\prime}, \beta\right\}$ in the figure is taken as $\{0.8770,8.4078\}$, when $N=50$, that the model can match Planck + WMAP9+ The $95 \%$ CL of the BAO data is compatible. When $N=60$, the model can be compatible with the $95 \%$ CL of the Planck + WMAP9 + BAO data.

The Fig. 2 shows three curves with different parameters. Since the curve is from Eq. (57):

$r=\frac{A_{T}^{2}}{A_{s}^{2}}=\frac{8 \kappa^{4}}{75 k^{3} C} \frac{V^{2}(\sqrt{1+K f V}-1)}{V^{\prime 2} f}$ 
Fig. 2 Relationships between the tensor-scalar ratio $r$ and the spectral index $n_{s}$. The orange, green, blue lines correspond to parameters, $\left\{\sigma^{\prime}, \beta\right\}$ $=\{1.1405,7.3919\}$, $\{1.1141,1.0673\}$, $\{0.8770,8.4078\}$ respectively for $V(\varphi)=\frac{\sigma}{2} \varphi^{2}$

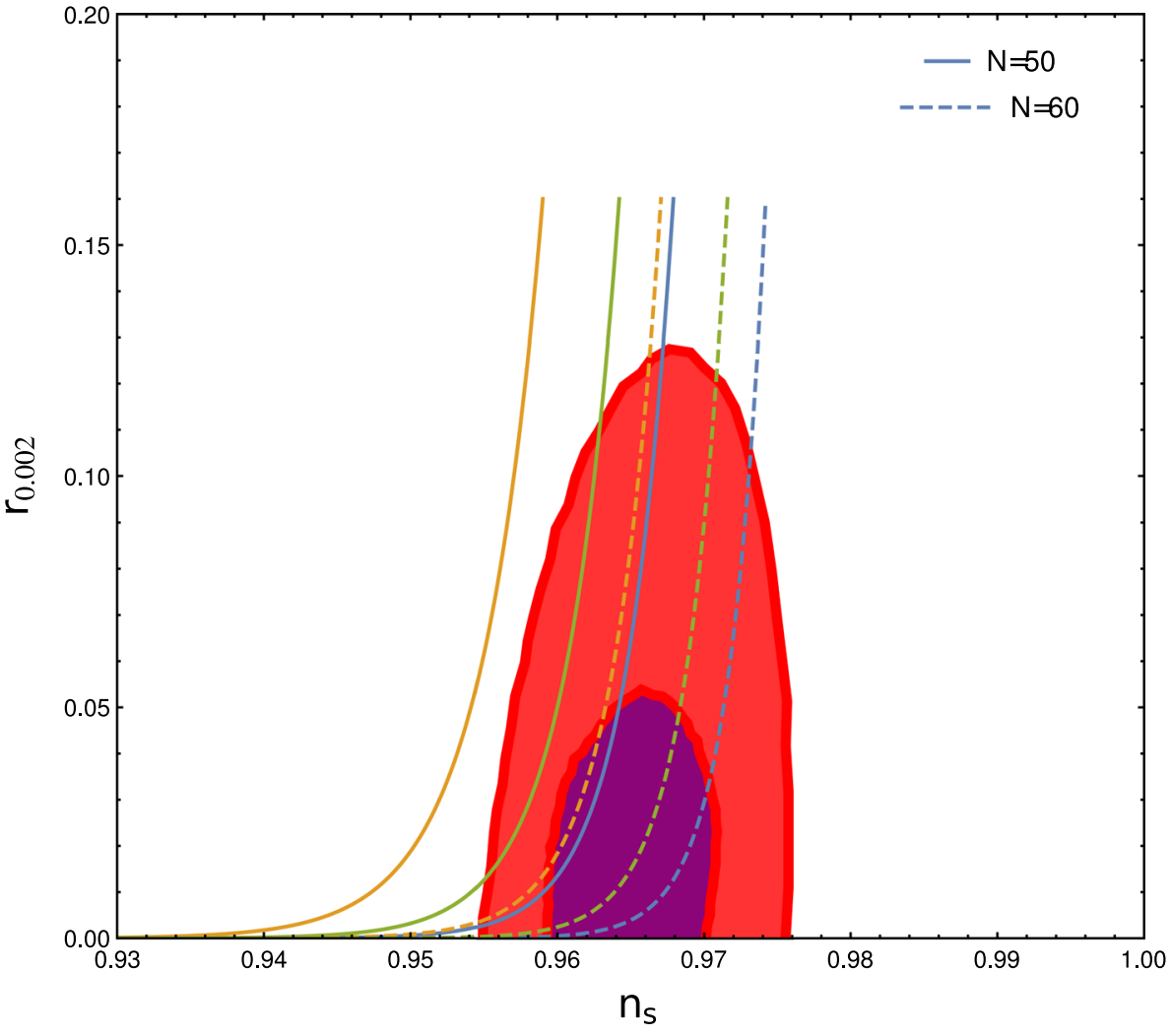

$$
\begin{aligned}
& \times \exp \left(-\int\left[\frac{V^{\prime}}{V}-\frac{V^{\prime \prime}}{V^{\prime}}\right.\right. \\
& \left.\left.+\frac{\mathcal{K}}{-f^{\prime} f^{-2} \sqrt{1+K f V}+f^{\prime} f^{-2}+\frac{1}{2} K f^{\prime} f^{-1} V-\frac{1}{2} K V^{\prime}}\right] d \varphi\right),
\end{aligned}
$$

i.e.

$r=\frac{8 \kappa^{4}}{75 k^{3} C} \frac{V^{2} \sqrt{1+f V}}{V^{\prime 2}} \exp \left(-3 \int H^{2}\left(n_{s}-1\right) d \varphi\right)$.

Therefore, we can see that $r$ and $n_{s}$ are exponentially related.

$\mathrm{B}: V(\varphi)=\frac{\sigma}{4} \varphi^{4}$

In the second step, we consider the quartic potential $V(\varphi)=\frac{\sigma}{4} \varphi^{4}$.

We introduce $\epsilon \equiv \frac{\dot{H}}{H}=1$ (corresponding to the end of the inflation), and we get $\varphi_{f}$. Then, by substituting the result into $N=\int_{t_{h c}}^{t_{f}} H d t$, we get $\varphi_{h c}$. By substituting $\varphi_{h c}$ into $n_{s}-1$ and $r=\frac{A_{T}^{2}}{A_{s}^{2}}$, we draw a relationship between the tensorscalar ratio and the spectral index. In the context of Planck + WMAP9 + BAO joint data, the following figure is drawn for $N=50$ and $N=60$ (Fig. 3).

In the Fig. 3, parameter group $\left\{\sigma^{\prime}, \beta\right\}$ takes $\{0.0023$, $3.5974\}$, the model is at $N=50$,it is not compatible with Planck + WMAP9 + BAO data. When $N=60$, the model can be compatible with $95 \%$ CL of Planck + WMAP9 +
BAO data. When the parameter group $\left\{\sigma^{\prime}, \beta\right\}$ is taken as $\{0.0029,7.7111\}$ in the figure, when $N=50$, that the model can match Planck + WMAP9 + 67\% CL of BAO data is compatible, and the model can be compatible with Planck + WMAP9 + 95\% CL of BAO data when $N=60$.

The Fig. 3 shows two curves with different parameters. Since the curve is from Eq. (57):

$$
\begin{aligned}
r= & \frac{A_{T}^{2}}{A_{s}^{2}}=\frac{8 \kappa^{4}}{75 k^{3} C} \frac{V^{2}(\sqrt{1+K f V}-1)}{V^{\prime 2} f} \\
& \times \exp \left(-\int\left[\frac{V^{\prime}}{V}-\frac{V^{\prime \prime}}{V^{\prime}}\right.\right. \\
& \left.\left.+\frac{\mathcal{K}}{-f^{\prime} f^{-2} \sqrt{1+K f V}+f^{\prime} f^{-2}+\frac{1}{2} K f^{\prime} f^{-1} V-\frac{1}{2} K V^{\prime}}\right] d \varphi\right),
\end{aligned}
$$

i.e.

$r=\frac{8 \kappa^{4}}{75 k^{3} C} \frac{V^{2} \sqrt{1+f V}}{V^{\prime 2}} \exp \left(-3 \int H^{2}\left(n_{s}-1\right) d \varphi\right)$

Therefore, we can see that $r$ and $n_{s}-1$ are exponentially related.

\section{Discussion}

The puzzle of the conversion of kinetic and potential energy in equal rights is the puzzle of the conversion, continuing to 
Fig. 3 Relationships between the tensor-scalar ratio $r$ and the spectral index $n_{s}$. The orange, blue lines correspond to parameters $\left\{\sigma^{\prime}, \beta\right\}=$ $\{0.0023,3.5974\}$, $\{0.0029,7.7111\}$, respectively for $V(\varphi)=\frac{\sigma}{4} \varphi^{4}$

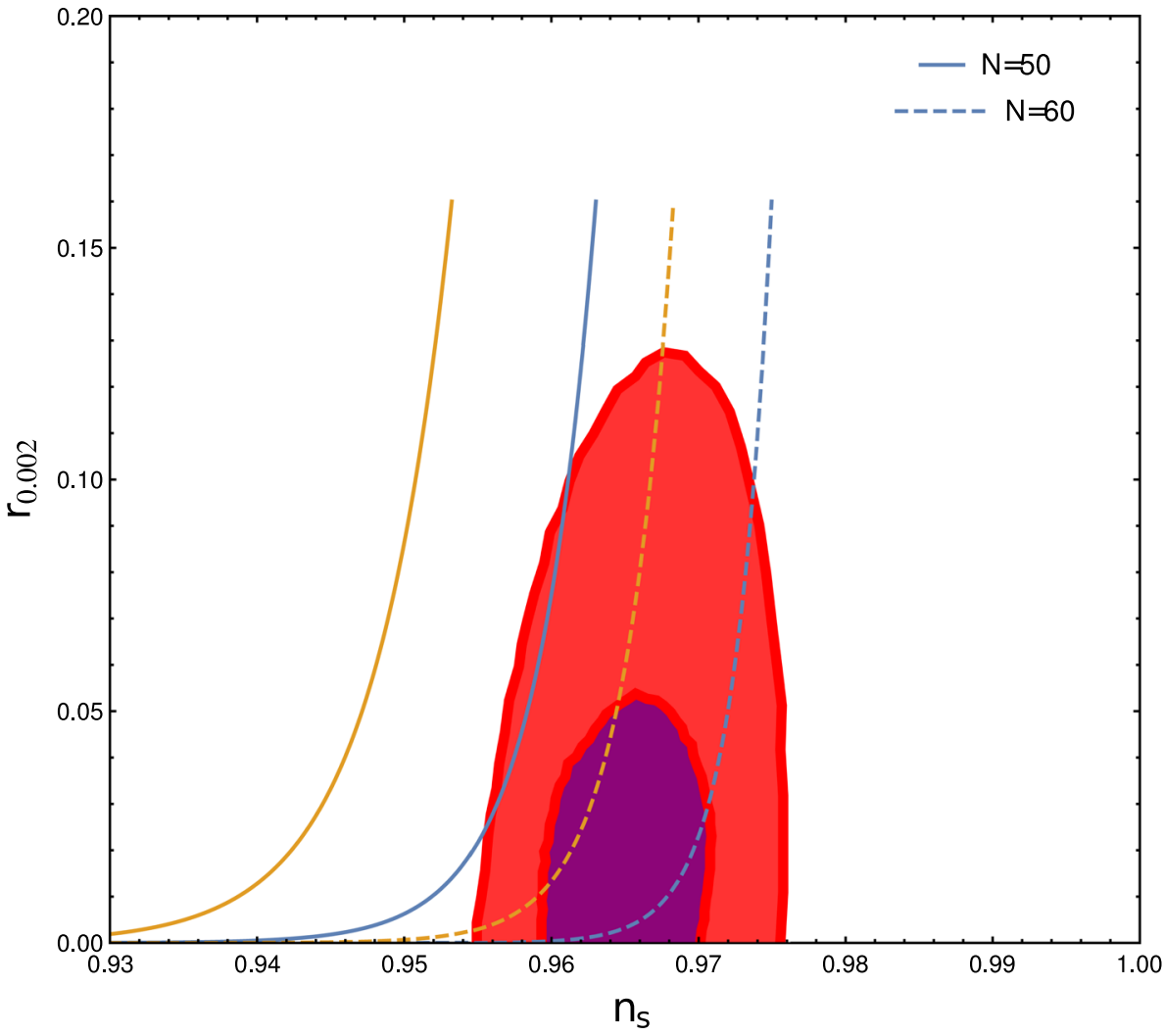

the end of the inflation of the universe, of potential energy and kinetic energy each other in these current DBI actions ( e.g., in the literature $[26,33,34]$ ), which can only be carried out under the first order approximation, and the potential energy cannot be completely converted to kinetic energy under any highorder, e.g., see Eq. (21) and the relative discussions below Eq. (20) in Sect. 2.

The inflation is a period of exponential supercooled expansion and leads to a universe consistent with observed spatial isotropy and homogeneity, and the relatively low temperature is maintained during the inflationary phase [62] ( which means that as usual there is the conversion of kinetic and potential energy), because of after the end of the inflation, the inflaton (e.g., scalar field, and a true vacuum is filled with a large number of inflaton particles ) leading to the inflation of the universe decays into some particles in great unification theory (i.e., in true vacuum after the inflation, inflaton particles decay, eventually giving rise to the observed Standard Model particles ), starting the radiation dominated phase of the universe, so that the universe is reheated.

The reheating after the inflation is divided as three phases, the first phase is called as preheating phase [63]. After the inflation, the energy in the action of this paper can also transfers from potential energy to regular matter energy (containing both kinetic and potential energy) during the preheating phase at the end of inflation, i.e., very closing after the end of the inflation [63]. The energy transfer process after the inflation of the universe has been well understood since the pioneering works $[62,63]$, so we don't repeat here. Especially, the action of this paper (in which the potential energy and kinetic energy are in the symmetric situation of equal right, which is key for a improved action ) not only produces the inflation, but also gives enough original density perturbations to form the universe we see today. Furthermore, all applications of the action of this paper are conforming to all the relative theories $[26,33,34]$ and all the current experiments $[26,33,34,62,63]$.

When establishing this new DBI action, we consider that the field that promotes the inflation of the universe exists in a $(1+3)$ dimensional spacetime and a 6-dimensional curly spacetime $[17,18,45,46,48]$. And regarding the $(1+3)$ radial $\mathrm{r}$ and passing through the 6-dimensional curly spacetime $[17,18,45,46,48]$. Then the corresponding line element can be expressed as Eq. (2) [22,44-46].

For the source of the scalar field, string theory gives an explanation, that is, the scalar field comes from the performance of the other dimensions beyond the four- dimensional spacetime in string theory. Assuming that Ads5 space is bent into a throat-like area, we pay attention to the moving D3brane in this area, and take the radial direction coordinate of the D3-brane as a scalar field [26,33,34]. If the space Ads5 itself is also moving, the throat-like area will also be deformed, which will affect the moving D3-brane [44-46]. At the same time the radial coordinates of the D3-brane must also change accordingly, which forms the movement of the dimensional world volume as a particle migrating along the 
scalar field and is affected by the distortion of the throat $[26,33,34,44-46]$. The action is related to the scalar field constructed in this way, which is called the DBI action [4446].

Because this action is the DBI action of D3-brane of the $(1+3)$-dimensional world volume in 10-dimensional string theory, drives the inflation of the universe and is not the action of deducing the four fundamental interactions in string theory [44], the background metric chosen here is not a ground state of string theory of deducing four fundamental interactions [44].

Because this paper proposes a true theory of the inflation, which overcomes all the difficulties of all the current DBI inflation theories (e.g., the potential energy and kinetic energy are not in the symmetric situation of equal right, which is key for a general action) [44,62], and conforms to all the relative theories and all the current experiments. Consequently, the theory of this paper is not simply another toy model of inflation, and adds useful physics and new physics meanings not only in string theory and but also in cosmology.

\section{Conclusion}

In this article, we deduce a symmetrical DBI action in equal rights for kinetic energy and potential energy in string theory, and introduce it into early cosmology, and calculate the inflation parameters and original density perturbations in detail. We deduce the influence of the scalar itself on the amplitude of the density perturbation, so that when different curl factors and potential functions are introduced, we can see the consistent results of the scalar field participating in the process of forming the perturbation. We see that because the scalar field is local, the tensor-scalar ratio is approximate to an exponential function. The parameters $\left\{\sigma^{\prime}, \beta\right\}$ brought by the curl factor and the potential function affect the shape of the exponential function, thereby we find that these lines relative to the parameters pass through the range defined by the current observation data, which means that the theory of this paper conforms to the current experiments.

Therefore, the symmetrical DBI action is important in string theory, produces the inflation, and it yields enough original density perturbations to form the universe we see today. The symmetrical DBI action solve the current general DBI action puzzle of the conversion of kinetic energy and potential energy in equal rights in string theory, and it predicts the power spectrum of satisfying the current observations, thus showing that the theory of this paper is consistent, supplementing the existing theories, and also a new way of explaining the inflation of the universe.

Acknowledgements The work is supported by National Natural Science Foundation of China (No. 11875081).
Data Availability Statement This manuscript has no associated data or the data will not be deposited. [Authors' comment: All numerical results and initial value is written in the article by some analytical formulas. Thus the data is not deposited.]

Open Access This article is licensed under a Creative Commons Attribution 4.0 International License, which permits use, sharing, adaptation, distribution and reproduction in any medium or format, as long as you give appropriate credit to the original author(s) and the source, provide a link to the Creative Commons licence, and indicate if changes were made. The images or other third party material in this article are included in the article's Creative Commons licence, unless indicated otherwise in a credit line to the material. If material is not included in the article's Creative Commons licence and your intended use is not permitted by statutory regulation or exceeds the permitted use, you will need to obtain permission directly from the copyright holder. To view a copy of this licence, visit http://creativecomm ons.org/licenses/by/4.0/.

Funded by $\mathrm{SCOAP}^{3}$.

\section{References}

1. D.N. Spergel et al., WMAP. Astrophys. J. Suppl. 170, 377 (2007). https://doi.org/10.1086/513700 arXiv:astro-ph/0603449

2. L. Alabidi, D. Lyth, JCAP 08, 013 (2006). https://doi.org/10.1088/ 1475-7516/2006/08/013 arXiv:astro-ph/0603539

3. U. Seljak, A. Slosar, P. McDonald, JCAP 10, 014 (2006). https://doi.org/10.1088/1475-7516/2006/10/014 arXiv:astro-ph/0603539

4. W.H. Kinney, E.W. Kolb, A. Melchiorri, A. Riotto, Phys. Rev. D 74, 023502 (2006). https://doi.org/10.1103/PhysRevD.74.023502 arXiv:astro-ph/0605338

5. S. Cole et al. [2dFGRS Collaboration], Mon. Not. R. Astron. Soc. 362, 505 (2005). https://doi.org/10.1111/j.1365-2966.2005. 09318.x

6. V. Barger, H.S. Lee, D. Marfatia, Phys. Lett. B 565, 33 (2003). https://doi.org/10.1016/S0370-2693(03)00757-3 arXiv:hep-ph/0302150

7. A.H. Guth, Phys. Rev. D 23, 347 (1981). https://doi.org/10.1103/ PhysRevD.23.347

8. A.H. Guth, Adv. Ser. Astrophys. Cosmol. 3, 139 (1987)

9. A.D. Linde, Phys. Lett. 108B, 389 (1982). https://doi.org/10.1016/ 0370-2693(82)91219-9

10. A.D. Linde, Adv. Ser. Astrophys. Cosmol. 3, 149 (1987)

11. Fayyazuddin, O.H. Mobarek, Phys. Rev. D 48, 1220 (1993). https:// doi.org/10.1103/PhysRevD.48.1220

12. I. Debono, arXiv:2103.03291 [astro-ph.CO]

13. J.E. Lidsey, A.R. Liddle, E.W. Kolb, E.J. Copeland, T. Barreiro, M. Abney, Rev. Mod. Phys. 69, 373 (1997). https://doi.org/10.1103/ RevModPhys.69.373 arXiv:astro-ph/9508078

14. A.D. Linde, Contemp. Concepts Phys. 5, 1-362 (1990)

15. A. Riotto, arXiv: 1010.2642 [hep-ph]

16. A.A. Starobinsky, Phys. Lett. B 91, 99-102 (1980). https://doi.org/ 10.1016/0370-2693(80)90670-X

17. A. Albrecht, P.J. Steinhardt, Phys. Rev. Lett. 48, 1220 (1982)

18. A. Albrecht, P.J. Steinhardt, Adv. Ser. Astrophys. Cosmol. 3, 158 (1987). https://doi.org/10.1103/PhysRevLett.48.1220

19. G.R. Dvali, S.H.H. Tye, Phys. Lett. B 450, 72 (1999). https://doi. org/10.1016/S0370-2693(99)00132-X arXiv:hep-ph/9812483

20. S. Kachru, R. Kallosh, A.D. Linde, J.M. Maldacena, L.P. McAllister, S.P. Trivedi, JCAP 0310, 013 (2003). https://doi.org/10.1088/ 1475-7516/2003/10/013 arXiv:hep-th/0308055 
21. X. Chen, M. Huang, S. Kachru, G. Shiu, JCAP 0701, 002 (2007). https://doi.org/10.1088/1475-7516/2007/01/002. arXiv:hep-th/0605045

22. C.H. Liu, S.T. Yau, arXiv:0907.0268 [math.AG]

23. W.H. Kinney, K. Tzirakis, Phys. Rev. D 77(2008). https://doi.org/ 10.1103/PhysRevD.77.103517. arXiv:0712.2043 [astro-ph]

24. A.J. Tolley, M. Wyman, JCAP 04, 028 (2008). https://doi.org/10. 1088/1475-7516/2008/04/028 arXiv:0801.1854 [hep-th]

25. H.V. Peiris, D. Baumann, B. Friedman, A. Cooray, Phys. Rev. D 76, 103517 (2007). https://doi.org/10.1103/PhysRevD.76.103517 arXiv:0706.1240 [astro-ph]

26. C. Armendariz-Picon, T. Damour, V.F. Mukhanov, Phys. Lett. B 458, 209-218 (1999). https://doi.org/10.1016/ S0370-2693(99)00603-6 arXiv:hep-th/9904075

27. J. Garriga, V.F. Mukhanov, Phys. Lett. B 458, 219-225 (1999). https://doi.org/10.1016/S0370-2693(99)00602-4 arXiv:hep-th/9904176

28. S.E. Shandera, S.H.H. Tye, JCAP 05, 007 (2006). https://doi.org/ 10.1088/1475-7516/2006/05/007 arXiv:hep-th/0601099

29. M. Alishahiha, E. Silverstein, D. Tong, Phys. Rev. D 70, 123505 (2004). https://doi.org/10.1103/PhysRevD.70.123505 arXiv:hep-th/0404084

30. E. Silverstein, D. Tong, Phys. Rev. D 70, 103505 (2004). https:// doi.org/10.1103/PhysRevD.70.103505 arXiv:hep-th/0310221

31. X. Chen, M. Huang, G. Shiu, Phys. Rev. D 74, 121301 (2006). $\quad$ https://doi.org/10.1103/PhysRevD.74.121301 arXiv:hep-th/0610235

32. J.M. Maldacena, JHEP 0305, 013 (2003). https://doi.org/10.1088/ 1126-6708/2003/05/013 arXiv:astro-ph/0210603

33. R. Bean, S.E. Shandera, S.H. Henry Tye, J. Xu, JCAP 0705, 004 (2007). https://doi.org/10.1088/1475-7516/2007/05/ 004. arXiv:hep-th/0702107

34. J.E. Lidsey, I. Huston, JCAP 0707, 002 (2007). https://doi.org/10. 1088/1475-7516/2007/07/002 arXiv:0705.0240 [hep-th]

35. G. Goon, S. Melville, J. Nollerin, Quantum corrections to generic branes: DBI, NLSM, and more. J. High Energy Phys. 1, 159 (2021)

36. P. Nath, M. Piskunovin, Supersymmetric Dirac-Born-Infeld axionic inflation and non-Gaussianity. J. High Energy Phys. 2, 034 (2019)

37. Z. Yinin, The infrared structure of exceptional scalar theories. J. High Energy Phys. 3, 158 (2019)

38. T. Grall, S. Jazayeri, D. Stefanyszynin, The cosmological phonon: symmetries and amplitudes on sub-horizon scales. J. High Energy Phys. 11, 097 (2020)

39. I. Antoniadis, H. Jiang, O. Lacombein, Note on supersymmetric Dirac-Born-Infeld action with Fayet-Iliopoulos term. J. High Energy Phys. 5, 111 (2020)

40. S. Aoki, Y. Yamadain, More on DBI action in 4D N $=1$ supergravity. J. High Energy Phys. 1, 121 (2017)

41. S. Bielleman, L.E. Ibanez, F.G. Pedro, I. Valenzuela, C. Wieck, JHEP 05, 095 (2016). https://doi.org/10.1007/JHEP05(2016)095 arXiv:0705.0240 [hep-th]

42. W.H. Kinney, E.W. Kolb, A. Melchiorri, A. Riotto, Inflation model constraints from the Wilkinson Microwave Anisotropy Probe threeyear data. Phys. Rev. D 74, 023502 (2006). arXiv:astro-ph/0605338 [INSPIRE]
43. E. Kahya, R. Woodard, Scalar field equations from quantum gravity during inflation. Phys. Rev. D 77, 084012 (2008)

44. K. Becker, M. Becker, J.H. Schwarz, String Theory and M-Theory (Cambridge University Press, Cambridge, 2006)

45. H. Firouzjahi, S.H.H. Tye, JCAP 03, 009 (2005). https://doi.org/ 10.1088/1475-7516/2005/03/009 arXiv:hep-th/0501099

46. M. Spalinski, JCAP 04, 018 (2007). https://doi.org/10.1088/ 1475-7516/2007/04/018 arXiv:hep-th/0702118

47. K. Nozari, N. Rashidi, Phys. Rev. D 88(8) (2013). https://doi.org/ 10.1103/PhysRevD.88.084040. arXiv:1310.3989 [astro-ph.CO]

48. J.M. Bardeen, Phys. Rev. D 22, 1882-1905 (1980). https://doi.org/ 10.1103/PhysRevD.22.1882

49. V.F. Mukhanov, H.A. Feldman, R.H. Brandenberger, Phys. Rep. 215, 203 (1992). https://doi.org/10.1016/0370-1573(92)90044-Z

50. E. Bertschinger, arXiv:astro-ph/9503125

51. L. Amendola, C. Charmousis, S.C. Davis, JCAP 0612 , 020 (2006). https://doi.org/10.1088/1475-7516/2006/12/020 arXiv:hep-th/0506137

52. L. Amendola, C. Charmousis, S.C. Davis, JCAP 0710, 004 (2007). https://doi.org/10.1088/1475-7516/2007/10/004 arXiv:0704.0175 [astro-ph]

53. K. Nozari, N. Rashidi, Phys. Rev. D 86, 043505 (2012). https://doi. org/10.1103/PhysRevD.86.043505 arXiv:1207.3966 [gr-qc]

54. K. Nozari, N. Rashidi, Phys. Rev. D 88(2) (2013). https://doi.org/ 10.1103/PhysRevD.88.023519. arXiv:1306.5853 [gr-qc]

55. B.A. Bassett, F. Tamburini, D.I. Kaiser, R. Maartens, Nucl. Phys. B 561, 188 (1999). https://doi.org/10.1016/S0550-3213(99)00495-2 arXiv:hep-ph/9901319

56. C. Gordon, D. Wands, B.A. Bassett, R. Maartens, Phys. Rev. D 63, 023506 (2000). https://doi.org/10.1103/PhysRevD.63.023506 arXiv:astro-ph/0009131

57. N. Kaloper, Phys. Rev. D 71, 086003 (2005). [Erratum: Phys. Rev. D 71 (2005), 129905]. https://doi.org/10.1103/PhysRevD.71. 129905. arXiv:hep-th/0502035

58. R. Maartens, D. Wands, B.A. Bassett, I. Heard, Phys. Rev. D 62, 041301 (2000). https://doi.org/10.1103/PhysRevD.62.041301 arXiv:hep-ph/9912464

59. D. Langlois, R. Maartens, D. Wands, Phys. Lett. B 489, 259 (2000). https://doi.org/10.1016/S0370-2693(00)00957-6 arXiv:hep-th/0006007

60. D. Langlois, F. Vernizzi, JCAP 0702, 017 (2007). https://doi.org/ 10.1088/1475-7516/2007/02/017 arXiv:astro-ph/0610064

61. S. Tsujikawa, J. Ohashi, S. Kuroyanagi, A. De Felice, Phys. Rev. D 88(2) (2013). https://doi.org/10.1103/PhysRevD.88.023529. arXiv:1305.3044 [astro-ph.CO]

62. A.D. Linde, Particle Physics and Inflationary Cosmology (Harwood, Chur, 1990)

63. L. Kofman, A. Linde, A. Starobinsky, Reheating after inflation. Phys. Rev. Lett. 73, 3195-3198 (1994) 